\title{
Low acute insulin response to intravenous glucose. A sensitive but non-specific marker of early stages of Type 1 (insulin-dependent) diabetes
}

\author{
B.Vialettes, C.Mattei-Zevaco, C. Badier, G. Ramahandridona, V.Lassmann-Vague and Ph. Vague \\ Department of Diabetology, University of Marseille, France
}

\begin{abstract}
Summary. Preventive treatment of Type 1 (insulin-dependent) diabetes presupposes early and accurate diagnosis of prediabetic states. The low acute insulin response to intravenous glucose has been proposed as a marker of both pre-Type 1 and pre-Type 2 (non-insulin-dependent) diabetes. In order to test the reliability of this marker for clinical detection of Type 1 diabetes we looked for this anomaly in 150 first degree relatives of Type 1 diabetic patients, 31 relatives of Type 2 diabetic patients and 39 young non-obese diabetic patients with mild or transient hyperglycaemia. The low acute insulin response was defined by a peak insulin value (sum of plasma insulin at 2 and $5 \mathrm{~min}$ after glucose load, $0.3 \mathrm{~g} / \mathrm{kg}$ body weight) below $50 \mu \mathrm{U} / \mathrm{ml}$. It was observed in $12 \%$ of the relatives of Type 1 diabetic patients ( 2 of them became diabetic) and in $13 \%$ of the relatives of Type 2 diabetic patients. Reproducibility of the peak insulin
\end{abstract}

value in 2 subsequent tests $(r=0.749)$ was inadequate to interpret small variations in one individual. In the population of 39 diabetic patients, 10 subsequently developed typical Type 1 diabetes, 9 were low insulin responders. In the 29 patients who are still non-insulin-dependent 3 years later, the anomaly was found in the 3 islet cell antibody-positive subjects and 11 out of 26 patients with no detectable antibodies. In conclusion, low acute insulin response to glucose is a sensitive but non-specific marker of early stages of Type 1 diabetes as this anomaly is shared by both Type 2 and Type 1 diabetes.

Key words: Type 1 (insulin-dependent) diabetes, Type 2 (non-insulin-dependent) diabetes, insulin secretion, preType 1 diabetes, maturity onset diabetes in the young.
Findings from family studies of Type 1 (insulin-dependent) diabetes suggest that in many cases the fullfledged disease is preceded by a latency period in which pancreatic $B$ cells are irretrievably destroyed by an autoimmune process [1]. If a safe and efficient aetiologic therapy is ever developed, its use will depend on being able to identify those subjects highly prone to diabetes. Srikanta et al. showed that a progressive loss of first phase insulin secretion after intravenous glucose load occurs during the subclinical phase of diabetes [2]. The low acute insulin release could therefore serve as a metabolic marker for the detection of high risk patients for diabetes who might benefit from a hypothetical preventive therapy [3, 4].

Low acute insulin release is not, however, a totally specific marker. A decrease in first phase insulin release after glucose has also been cited as a characteristic feature in association with Type 2 (non-insulin-dependent) diabetes and genetic predisposition to that state [5-9]. Thus, the question arises as to whether the low acute in- sulin response is a reliable marker of pre-Type 1 diabetes in young patients and if preventive treatment, when developed, could be undertaken on that evidence alone. In order to evaluate the usefulness of this marker for early detection of Type 1 diabetes at the individual level, the early insulin response after hyperglycaemia induced by intravenous injection was studied with a single parameter in a population considered as high risk or in the early phase of Type 1 diabetes or Type 2 diabetes. This study was performed on non-diabetic subjects who had first degree relatives presenting Type 1 or Type 2 diabetes and on diabetic patients characterised by a mild or transient hyperglycaemia. The ages of these nonobese subjects ranged from 5 to 45 years, which is compatible with the age of onset of Type 1 diabetes. To further document the classification of diabetes, islet cell antibodies were investigated and patients were followed for three years. By this means we attempted to define the prevalence of low acute insulin response in clearcut situations leading to either Type 2 or Type 1 diabetes. 


\section{Subjects and methods}

\section{Subjects}

The body weight of all patients included in this study was normal; all of them had a body mass index below $27 \mathrm{~kg} / \mathrm{m}^{2}$. This age ranged from 5 to 45 years. The study was performed in accordance with the principles of the declaration of Helsinki. Each patient was informed of the reasons for the investigation and gave his oral consent. Four groups were studied as follows:

Control group. This group was composed of 67 subjects $(22.9 \pm 3$ years) with no family history of diabetes and normal glucose tolerance verified by a glucose assimilation coeficient, $\mathrm{K}$ value $>1.2 \times 10^{-2}$ after an i.v. injection of glucose.

Type 1 relatives group. This group was composed of 150 first degree relatives of Type 1 diabetic patients ( $15.8 \pm 0.8$ years). These subjects were recruited in a cohort investigated during a prospective family study undertaken in 1984 under a grant from the Institut National de la Santé et de la Recherche Médicale. All had normal plasma glucose levels ( $\leq 5.5 \mathrm{mmol} / \mathrm{l}$ ) on the day of examination. $\mathrm{K}$ values were not determined. This group included 79 siblings and 74 children of Type 1 diabetic patients. Sixteen were islet cell antibodies (ICA) positive. This incidence is not representative of the positive results recorded in the original cohort $(17 / 290 ; 5.86 \%)$.

Type 2 relatives group. This group was composed of 31 first degree relatives of Type 2 diabetic patients ( $20 \pm 2$ years) including 4 siblings and 27 children of long-duration Type 2 diabetic patients. All of them had normal glucose tolerance $\left(\mathrm{K}>1.20 \times 10^{-2}\right)$.

Diabetic group. This group was composed of 39 patients (33.3 \pm 1.7 years) with mild or transient hyperglycaemia acertained by two separate measurements of fasting plasma glucose level between 7.7 and $10 \mathrm{mmol} / 1$, occurring spontaneously or under diabetogenic conditions (treatment by corticoids, oral contraceptives, surgical stress). All these patients were checked for ICA and were kept under clinical observation for 3 years. Ten of them became insulin-dependent ( $5 / 10$ were ICA positive) and 3 were permanently ICA positive but remained controllable by diet and oral hypoglycaemic agents. The remaining 26 remained ICA negative and did not require insulin throughout the 3 -year observation period. They were presumed to be real Type 2 diabetic patients. Seventeen of these patients had a family history of long-standing Type 2 diabetes involving at least one first-degree relative or two second-degree relatives. Their conditions probably can be classified as maturity onset diabetes in the young (MODY) as described by Fajans et al. [10].

\section{Investigations}

Intravenous glucose tolerance tests were performed at 08.00 hours on patients resting supine after an overnight fasting period. If the patient was using hypoglycaemic agents, treatment was discontinued $48 \mathrm{~h}$ prior to testing. Plasma insulin was measured before and then 2 , 5 and $10 \mathrm{~min}$ after i.v. glucose load $(0.3 \mathrm{~g} / \mathrm{kg})$ injected within less than $2 \mathrm{~min}$. A detailed description of this technique and its rationale has been previously published elsewhere [7]. First phase insulin release was calculated as the sum of plasma insulin measured at 2 and 5 min after glucose load. Cytoplasmic type islet cell antibodies were detected by indirect immunofluorescence on a frozen section of human pancreas according to the method derived from that described by Bottazzo [11].

\section{Statistical analysis}

Descriptive statistics were performed using "Statgraphics statistical graphics System". Comparison between groups was performed using the Student's t-test.

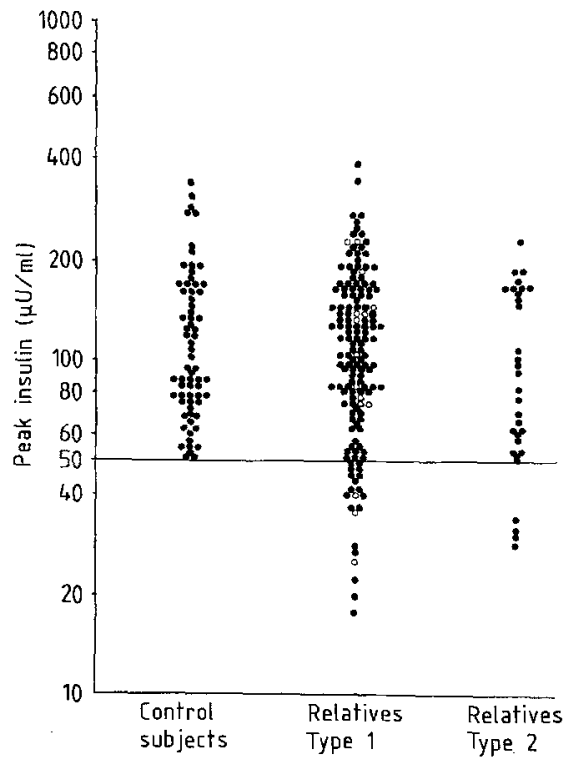

Fig.1. Peak insulin response to i.v. glucose in glucose tolerant populations: control subjects, first degree relatives of Type 1 diabetic patients, first degree relatives of Type 2 diabetic patients. Subjects who were positive for islet cell antibodies (o)

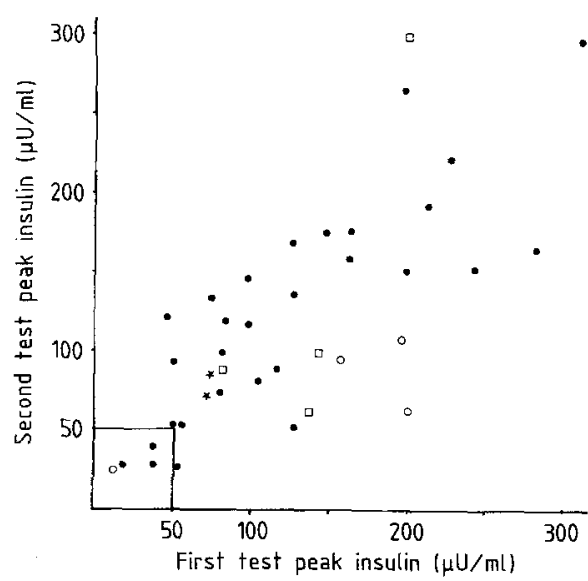

Fig. 2. Variations in peak insulin response to i.v. glucose measured at 6-month intervals in 39 first degree relatives of Type 1 diabetic patients. Symbols indicate changes in islet cell antibody status: - = consistently negative subjects; $\square=$ positive subjects who became negative; ${ }^{*}=$ consistently positive subjects: $O=$ negative subjects who became positive

\section{Results}

\section{Definition of the low acute insulin response to intravenous glucose}

The normal range of the peak insulin values (sum of plasma insulin at 2 and $5 \mathrm{~min}$ ) was established from data recorded in the normal population (Fig. 1). The distribution of the peak insulin value was normal after logarithm transformation. The mean calculated from log-transformed data was $108.14 \mu \mathrm{U} / \mathrm{ml}$ and $95 \%$ confidence limits, 41.9-284 $\mu \mathrm{U} / \mathrm{ml}$. Arbitrarily we defined the low acute insulin response by a value of peak insulin below the lowest response of the control group $(50 \mu \mathrm{U} / \mathrm{ml})$. After modelisation of the distribution, it 
appeared that this value corresponds to the 5.5 th percentile of the normal population. The 5 th percentile and the 10th percentile were, respectively, 48.81 and $58.19 \mu \mathrm{U} / \mathrm{ml}$.

Incidence of the low acute insulin-response in relatives of Type 2 diabetic patients

As a group, relatives of Type 2 diabetic patients presented a weaker insulin response than control subjects. The mean calculated from log-transformed data was $91.53 \mu \mathrm{U} / \mathrm{ml} \quad(95 \%$ confidence limits: $27.6-$ $302 \mu \mathrm{U} / \mathrm{ml}$. This difference was not statistically significant. In this group, 4 out of 31 subjects $(13 \%)$ had a peak insulin value lower than normal.

\section{Incidence of the low acute insulin response in relatives} of Type 1 diabetic patients

The response in this Type 1 relatives group was comparable to the one recorded in the control group (mean: $100.37 \mu \mathrm{U} / \mathrm{ml}, 95 \%$ confidence limits 30.9 $324.34 \mu \mathrm{U} / \mathrm{ml}$ (Fig. 1). A defective response was noted in $12 \%$ of cases. Out of the 150 relatives of Type 1 diabetic patients 16 were ICA positive and their insulin response was not different from ICA negative subjects. Three of them exhibited an absence of acute insulin response and one became diabetic 6 months later. Of the 134 ICA negative subjects, 15 exhibited absence of early response and one became diabetic.

In this group, 39 patients were tested twice at a 6month interval. The variations of the peak insulin values are shown in Figure 2. There was a correlation between these two values $(r=0.749, p<0.001)$. Two patients with a borderline value went through the $50 \mu \mathrm{U} / \mathrm{ml}$ limit for 6 months. Changes in ICA status from negative to positive or vice versa apparently had no influence on acute insulin response. In this group only one subject with permanent abnormal response became diabetic.

\section{Incidence of low acute insulin response in mildly or transiently hyperglycaemic diabetic subjects}

Individual peak insulin values recorded in diabetic patients are listed in Figure 3. In patients who became insulin-dependent later, low acute insulin response was almost consistently found $(9 / 10,37 \pm 2.38 \mu \mathrm{U} / \mathrm{ml}$, mean \pm SEM). The tenth subject (ICA negative) had a borderline peak insulin value of $51 \mu \mathrm{U} / \mathrm{ml}$ two years before becoming insulin-dependent. $\mathrm{He}$ was tested again 5 months before insulin dependence at which time the peak insulin value was unequivocally abnormal $(26 \mu \mathrm{U} / \mathrm{ml})$. Low acute insulin response was also observed in 3 subjects with detectable ICA. Despite the fact that they remained non-insulin-dependent

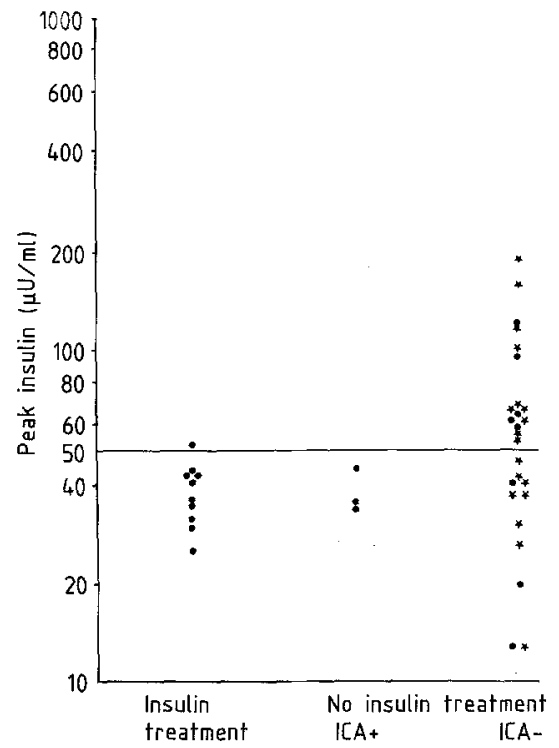

Fig.3. Peak insulin response to i.v. glucose in mildly or transiently diabetic patients according to the ICA status and the clinical evolution during the 3 following years. *Subjects with a family history of Type 2 diabetes

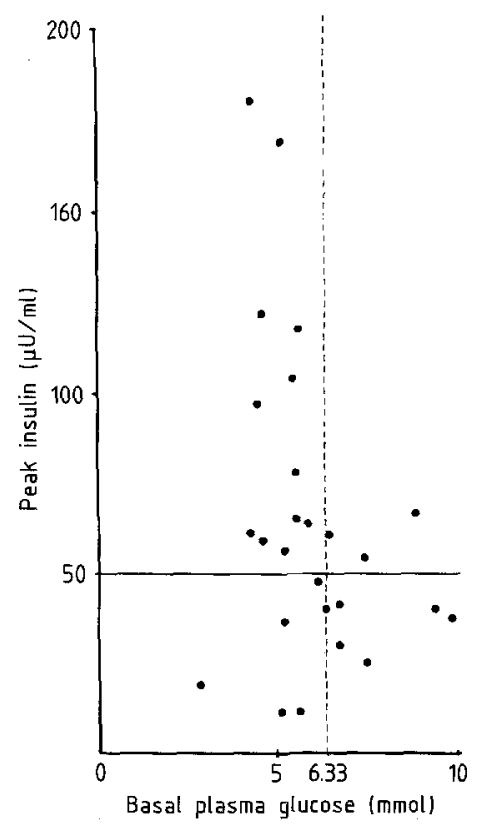

Fig.4. Relationship between peak insulin response to i.v. glucose and basal plasma glucose level on the day of testing in 26 subjects with mild or transient hyperglycaemia and no detectable islet cell antibody. None of them progressed to insulin-dependence during the 3 following years. The arbitrary limit of 6.33 was taken from Brunzell [15]

3 years later, these subjects could be considered to be suffering from slow Type 1 diabetes. Though less frequent, this anomaly has also been noted in hyperglycaemic subjects with no detectable ICA (11/26) who did not became insulin-dependent over the 3-year observation period $(55.9 \pm 6.08 \mu \mathrm{U} / \mathrm{ml})$. Seventeen of these subjects had a family history of Type 2 diabetes. Eight subjects in this subgroup (47\%) presented an ab- 
normal peak insulin response. To evaluate the relationship between basal glycaemia and acute insulin response, peak insulin was plotted as a function of actual fasting plasma glucose in the 26 ICA negative, still non-insulin-dependent diabetic subjects (Fig.4). A negative correlation $(r=-0.46 ; p<0.05$ ) was observed. In all but two subjects with fasting plasma glucose greater than $6.33 \mathrm{mmol} / 1(n=7)$, the peak insulin value was abnormal. However, five of the 18 subjects with nearly normal fasting glycaemia $(<6.33 \mathrm{mmol} / \mathrm{l})$ exhibited an absence of acute insulin response.

\section{Discussion}

If an aetiologic treatment of Type 1 diabetes was to become available, it would be important to have an investigative technique allowing accurate detection of the disease in the very early stages before insulin secretion stops. Since mass screening for preclinical diabetes in the general population is unfeasible at the present time, priority must be given to high risk populations. Previous studies have shown that first degree relatives of Type 1 diabetic patients and subjects with mild or transient hyperglycaemia are prime candidates for screening $[3,4,12,13]$. Low acute insulin release after i.v. glucose has been proposed as a metabolic marker of pre-Type 1 diabetes $[2,3]$. The purpose of this study was to evaluate the sensitivity and specificity of this marker in high risk patients. In the population studied, i.e. first degree relatives of diabetic patients and mildly hyperglycaemic diabetic subjects, this anomaly was permanently noted in subjects with true Type 1 diabetes. According to the chosen criteria, all relatives who became diabetic and almost all diabetic patients who became insulin-dependent were low insulin responders. Thus, it can be said that the sensitivity of this marker is excellent. However, as pointed out by Srikanta, it is a relatively late detector of the imminent failure of beta pancreatic activity [2]. In our series one mildly hyperglycaemic subject was still in borderline condition 2 years before insulin therapy was needed. A pathologic response was only recorded in tests carried out 5 months before this patient became insulin-dependent. The short term reproducibility of this test ( 6 months) seems relatively satisfactory to detect the abnormal response but inadequate to interpret variations in the normal range. The low reproducibility of this marker have been already reported by Smith [14] in normal subjects. In terms of specificity, this test rates much lower. Eleven percent of the first degree relatives of Type 1 diabetic patients without detectable ICA were low insulin responders. In this population the risk of developing diabetes in future years is low: in Srikanta's series [3] only 1 out of 1707 developed diabetes during the 2-year observation period $(<1 \%)$. In our population, 2 out of 290 developed diabetes over a 4-year period. If selection for preventive immu- nosuppressive therapy had been based on acute insulin response at least 9 out of 10 patients would have been unnecessarily treated. In the group composed of first degree relatives of Type 2 diabetic patients, low acute insulin response was observed in 4 out of 31 subjects. Given the fact that distinguishing between Type 1 and Type 2 diabetes in individual patients receiving insulin is sometimes difficult, it is obvious that the risk of wrongly treating a relative predisposed to Type 2 diabetes for Type 1 diabetes is high. The specificity of this test is even lower in subjects with very mild hyperglycaemia. In the group composed of mildly diabetic patients without islet cell antibodies, there was a negative correlation between basal plasma glucose and insulin response during the first $5 \mathrm{~min}$ of glucose stimulation. Buntzell [15] demonstrated that early insulin response disappears when plasma glucose exceeds $6.33 \mathrm{mmol} / \mathrm{l}$. In our series, among the subjects classified as Type 2 diabetic patients (criteria, ICA negative and non-insulin-dependent over the next 3 years), $28 \%$ of those with values below this threshold on the day of testing were low responders. Even in a subgroup of subjects who can be classified as suffering maturity onset diabetes in the young a proportion of $47 \%$ of low insulin responders was found. Absence of early insulin response after i.v. glucose load has been associated with both Type 1 and Type 2 diabetes. In the 1970s, Cerasi [5, 16], Fujita [8], Ratzmann [9], Vague [7, 17], and Varsano Aharon [6] confirmed that this qualitative anomaly in B-cell function was a common finding in Type 2 diabetes. According to Cerasi [16] and Vague [17] this feature is genetically transmitted as it is frequently found both in relatives of Type 2 diabetic patients and in subjects with impaired glucose tolerance. However, the criteria used to define low insulin response in these studies were so lax that anomaly was observed in no less than $17 \%$ of normal subjects $[5,16,17]$. Conceivably, stricter criteria would facilitate the distinction between subjects prone to Type 1 diabetes and those prone to Type 2 diabetes. Unfortunately, even with the criteria chosen in our study acute insulin response does not delineate Type 1 and Type 2 diabetes in populations selected with respect to either genetic predisposition or clinical onset of the disease.

It is likely that the mechanism underlying this functional anomaly common to both forms differs. For Type 1 diabetes, low insulin response after i.v. glucose probably results from a disease-related decrease in the insular mass while in Type 2 diabetes it probably corresponds to a defect in B-cell function. No method presently allows distinction between these two mechanisms.

In conclusion, because of its excellent sensitivity, low insulin response after i.v. glucose remains a valuable marker for early detection of Type 1 diabetes. However, in view of its lack of specificity, this test must be interpreted in conjunction with other markers of diabetes, especially immunologic markers. 
Acknowledgements. This study was supported by a grant of the Caisse Nationale de l'Assurance Maladie des Travailleurs Salariés, Paris.

\section{References}

1. Eisenbarth GS (1986) Type I Diabetes Mellitus, A chronic autoimmune disease. N Engl J Med 314: 1360-1368

2. Srikanta S, Ganda OP, Gleason RE, Jackson RA, Soeldner JS, Eisenbarth GS (1984) Pré-type I diabetes: linear loss of beta cell response to intravenous glucose. Diabetes 33: 713-720

3. Srikanta S, Ganda OP, Rabizadeh A, Soelner JS, Eisenbarth GS (1985) First degree relatives of patients with type I diabetes mellitus. Islet cell antibodies and abnormal insulin secretion. $\mathrm{N}$ Engl J Med 313: 461-464

4. Ginsberg-Fellner F, Witt ME, Franklin BM, Yagihashi $\mathrm{S}$, Togushi Y, Dobersen MS, Rubinstein P, Notkins AL (1985) Triad of markers for identifying children at risk of developing insulin-dependent diabetes mellitus. JAMA 254: 1469-1472

5. Cerasi E, Luft R. (1967) The plasma insulin response to gluocse infusion in health subjects and in diabetes mellitus. Acta Endocrinol (Kbh) 55: 278-334

6. Varsano Aharon N, Echemendia E, Yalow RS, Berson SA (1970) Early insulin response to glucose and to tolbutamide in maturity onset diabetes. Metabolism 19: 401-417

7. Vague Ph, Ramahandridona $\mathrm{G}$, Gerolami A (1974) Insulin response to glucose and to tolbutamide in essential versus "pancreatic" mild glucose intolerance. Diabetes 23: 896-901

8. Fujita Y, Herron AL, Selizer HS (1975) Confirmation of impaired early insulin response to glycaemic stimulus in non obese mild diabetes. Diabetes 24: 17-27

9. Ratzmann KP, Schultz B, Heinke P, Michaelis D (1981) Quantitative and qualitative changes in the early insulin response to glucose in subjects with impaired carbohydrate tolerance. Diabetes Care 4: 85-91
10. Fajans SS, Cloutier MC, Crowther RL (1979) Heterogeneity of idiopathic diabetes mellitus in man. In: Vague J, Vague $\mathrm{Ph}$ (eds) Diabetes and Obesity. Excerpta Medica, Amsterdam, pp 73-82

11. Vialettes B, Di Campo-Rougerie C, Lassmann V, Vague $\mathrm{Ph}$ (1983) Comparaison de divers types d'anticorps anti-ilots dans le diabète en fonction de la cause et de la durée d'évolution. Presse Médicale 12: 2303-2306

12. Gorsuch AN, Spencer KH, Lister J, Mc Nally JM, Dean BM, Bottazzo GF, Cudworth AG (1981) Evidence for a long prediabetic period in type I (insulin-dependent) diabetes mellitus. Lancet II: 1363-1365

13. Orchard TJ, Rosembloom AL (1985) The development of insulin-dependent diabetes mellitus among relatives. Diabetes Care 8 [Suppl]: $45-50$

14. Smith CP, Tarn AC, Thomas JM, Ovekamp D, Corakci A, Savage MO, Gale EAM (1988) Between and within subject variations of the first phase insulin response to intravenous glucose. Diabetologia 31: 123-125

15. Brunzell JD, Robertson RP, Lerner RL, Hazzard WR, Ensinck JW, Bierman EL, Porte D (1976) Relationships between fasting plasma glucose levels and insulin secretion during intravenous glucose tolerance test. J Clin Endocrinol Metab 42: 222-229

16. Cerasi E, Luft R (1967) What is inherited, what is added? Hypothesis for pathogenesis of diabetes mellitus. Diabetes 16: 615

17. Vague Ph, Ramahandridona G, Vague J (1975) Proportion de faibles secréteurs d'insuline parmi les descendants des diabétiques de l'age mur. Diabete Metab 1: 45-50

Received: 21 December 1987

and in revised form: 31 May 1988

Dr. B. Vialettes

Département de Diabétologie

Hôpital Universitaire Michel Lévy

84a, Rue de Lodi

F-13006 Marseille

France 